\title{
Krankheitsursachen im Wandel der Zeit
}

\section{René Bloch}

Buchtipp: Bloch R. Die Psychagogische Psychotherapie. Ein Denkmodell für das 21. Jahrhundert. Wien: Verlagshaus der Ärzte. 152 Seiten. 50.50 CHF. ISBN 978-3-99052-084-O

Korrespondenz:

Dr. med. René Bloch

Austrasse 7

CH-4106 Therwil

marc.girard8[at]wanadoo.fr
Seit Urzeiten obliegt dem Arzt die Aufgabe, Krankheiten zu heilen. Für den Mediziner aus vorwissenschaftlichen Epochen galt Krankheit als ein Geschehen, dessen Ursachen leicht auszumachen waren. Ihren äusseren Faktoren kam eine nur bescheidene Rolle zu, es sei denn im Falle gewaltsamer umweltbedingter oder dämonischer Einwirkungen. Die Krankheit wurde als störende Veränderung von Körperfunktionen und als Folge eines Missverhältnisses gewisser Körpersäfte angesehen. Nach und nach entstanden Krankheitslehren, die die Ursachen genauer zu differenzieren vermochten bis zu den Klassifikationssystemen, die wir seit der jüngeren Vergangenheit der Menschheitsgeschichte kennen. Durch diese Systeme können die Krankheiten in verschiedene Kategorien eingeteilt werden, je nach deren Erscheinungsformen oder Kenntnis über deren Ursprung.

Für eine ätiologische Einteilung werden die meisten Krankheitsursachen Dysfunktionen innerhalb des menschlichen Organismus zugeschrieben und zuletzt auch genetischen Anomalien. Hinzu kommen Krankheiten durch diverse äussere Einwirkungen, die sich in der natürlichen Umwelt des Menschen befinden. In letzter Zeit spielen Noxen eine Rolle, die durch von Menschen verursachte Veränderungen der Natur und des sozialen Gewebes zustande kommen.

Während früher die ursachenorientierte Klassifikation von Krankheiten darauf ausgerichtet war, Krankheitseinheiten zu beschreiben, die auf natürliche äussere pathogene Faktoren zurückzuführen wären, müsste man heute neue Kategorien von Krankheiten beschreiben, deren Ursachen nicht ursprünglich in der Umwelt vorkommen. Dieselben werden durch Eingriffe des Menschen selbst in dessen Umwelt erzeugt und den damit verbundenen multiplen ökologischen Veränderungen und Gleichgewichtsstörungen, ebenso gesellschaftliche Umstrukturierungen, die den Bedürfnissen des Menschen entgegengestellt sind.

Durch die Vielzahl zivilisationsbedingter Faktoren, welche die Gesundheit des Menschen in seiner physischen und psychischen Ganzheit bedrohen, bekommt der Arzt eine Aufgabe, die weit über das Tätigkeitsfeld hinausreicht, womit er sich noch vor einigen Jahrzehnten begnügen durfte. Weniger die individuelle Belastbarkeit als vielmehr die Belastungsfaktoren in der Umwelt schieben sich in den Mittelpunkt des diagnostischen In- teresses. Folglich kann die Therapie des Patienten heute nur noch zum Ziele führen, insofern die Tatsache erkannt wird, dass der Mensch selbst die Verursachung einer Vielzahl krankhafter Störungen psychophysischer Art verschuldet.

Das Ziel jeder Medizin ist die Heilung des Patienten durch Entfernung der krankmachenden Einwirkung. In der modernen Zeit könnte der Arzt dieser Aufgabe nur noch gerecht werden, indem er die Gesellschaft beeinflusst. Gelingt es nicht, die eigentlichen Krankheitsursachen und deren Abstammung zu erkennen, d.h. die Zerstörung der natürlichen Umwelt im Gefolge der technischen Entwicklung und die Schaffung einer artifiziellen Gesellschaftsform, muss man sich mit einer palliativen Therapie begnügen und damit rechnen, dass die krankhaften Störungen in der Bevölkerung weiter zunehmen, wobei ein rascher Anstieg der exogenen Krankheitsbilder heute schon erwiesen ist.

Das Aktionsfeld des Arztes wird durch die beschriebene Situation wesentlich erweitert, indem er seinen therapeutischen Einfluss über den Patienten hinaus auf dessen Umgebung richten muss. Dabei soll zunächst die Bewusstheit des Patienten für die komplexe Interaktion Mensch-Umwelt erreicht werden. Dem Einfluss des Arztes sind aber bei der Behandlung äusserer schädlicher Faktoren enge Grenzen gesetzt, da der einzelne Patient nicht für das Fehlverhalten der ganzen Gesellschaft verantwortlich gemacht werden kann. Eine Komplizität des Patienten am Fehlverhalten der Gesellschaft kann jedoch aufgedeckt und erklärt werden mit entsprechenden Vorschlägen für konstruktive Konsequenzen. Über einen solchen Rahmen hinaus bedarf es aber der Zusammenarbeit der Ärzte auf einem höheren Niveau, d.h. internationaler Standesorganisationen.

Es ist dringend notwendig, dass Arbeitsgruppen von Ärzten gebildet werden, die sich mit den vom Menschen selbst verursachten pathogenen Umweltfaktoren befassen, zur Ausarbeitung von Vorschlägen, um eine weitere Gefährdung der menschlichen Gesundheit weltweit zu verhüten. Eine Reform und Umkehr der gegenwärtigen Situation, die mit grösster Wahrscheinlichkeit zu weiteren destruktiven Veränderungen und ihren gesundheitsschädlichen Folgen führen wird, benötigt viel Mut und ein Umdenken auch in ethischen Fragen, deren Beantwortung uns heute leichtzufallen scheint. 